\section{Reply to Cipriani et al}

European Journal of Human Genetics (2012) 20, 2-3; doi:10.1038/ejhg.2011.177; published online 12 October 2011

In our 2009 paper entitled 'Variation near complement factor I is associated with risk of advanced AMD', ${ }^{1}$ we reported that variants across a wide region of chromosome 4 spanning a $173-\mathrm{kb}$ region are associated with age-related macular degeneration (AMD). Recently, we have conducted a genome-wide association study (GWAS) metaanalysis using imputed data derived from 2594 DNA samples from individuals with advanced AMD and 4134 control samples with European ancestry. ${ }^{2}$ In this sample, rs10033900 is the most strongly associated single nucleotide polymorphism (SNP) in the region to advanced AMD with a $P$-value of $2.4 \times 10^{-11}(\mathrm{OR}=0.76$; confidence interval $(\mathrm{CI})=0.70-0.82$; C-allele $=47.9 \%$ cases and $53.7 \%$ controls). In our original paper, ${ }^{1}$ we were unable to find a functional SNP in the $3^{\prime}$ exonic region of complement factor I or all four exonic regions of phospholipase $\mathrm{A}(2)$ group 12A (PLA2G12A) and the functional relevance of rs 10033900 is yet to be determined. Cipriani et $a^{3}$ have recently genotyped rs10033900 and did not find evidence for an association. While this is interesting, it is not very informative as lack of an association could be due to a variety of factors including chance, power, ascertainment heterogeneity, or population genetic heterogeneity.

Cipriani et $a l^{3}$ state that in their cohort consisting of 859 cases (96\% advanced AMD) and 423 controls, there is no association to the rs 10033900 SNP. However, this study configuration is not comparable in power to our studies. Using http://pngu.mgh.harvard.edu/ purcell/ gpc/cc2.html to compare sample sets, we can calculate that our original sample set had nearly twice the power of their discovery cohort and our meta-GWAS dataset has over five times their power. Cipriani et al $^{3}$ followed-up their finding in a second cohort of 505 cases (48\% advanced AMD) and 351 controls. As we used samples from only advanced cases of AMD for our analysis, and they included about 50\% non-advanced cases in their follow-up cohort, their analysis is not comparable to ours. Evaluation of the 244 advanced cases in their second cohort would yield less than half the power as their original cohort. As discovery samples often overestimate the effect size due to 'winner's curse', it is not surprising for smaller studies to not strongly replicate true findings. However, the estimated effect in both cohorts reported by Cipriani et $a^{3}$ are in the same direction as our observed effect, and their reported CIs overlap the CI from our large GWAS meta-analysis.

\section{CONFLICT OF INTEREST}

Massachusetts General Hospital (MJ Daly) and Tufts Medical Center (JM Seddon) have filed patent applications related to material presented.

Jesen A Fagerness ${ }^{1,2}$, Yi Yu ${ }^{3}$, Julian B Maller ${ }^{1,2,4}$, Benjamin M Neale ${ }^{1,2}$, Robyn C Reynolds ${ }^{3}$, Mark J Daly ${ }^{1,2}$ and Johanna M Seddon ${ }^{3,5}$

${ }^{1}$ Center for Human Genetic Research, Massachusetts General Hospital, Boston, MA, USA;

${ }^{2}$ Program in Medical and Population Genetics, Broad Institute of Harvard and MIT, Cambridge, MA, USA; ${ }^{3}$ Ophthalmic Epidemiology and Genetics Service, New England Eye Center, Tufts Medical Center, Boston, MA, USA;

${ }^{4}$ Department of Statistics, University of Oxford, Oxford, UK; ${ }^{5}$ Tufts University School of Medicine, Department of Ophthalmology, Boston, MA, USA E-mail: jseddon@tuftsmedicalcenter.org

1 Fagerness JA, Maller JB, Neale BM et al: Variation near complement factor $\mathrm{I}$ is associated with risk of advanced AMD. Eur J Hum Genet 2009; 17: 100-104.

2 Yu Y, Bhangale TR, Fagerness J et al: Common variants near FRK/COL1OA1 and VEGFA are associated with advanced age-related macular degeneration. Hum Mol Genetics 2011; 20: 3699-3709.

3 Cipriani V, Matharu BK, Khan JC et al: No evidence of association between complement factor I genetic variant rs 10033900 and age-related macular degeneration. Eur J Hum Genet 2011; 20: 1-2. 